\title{
Las nuevas políticas: repartos de poder, activismo y liderazgos en la PAH. Estudio de caso sobre el activismo juvenil en Lleida
}

\author{
Eduard Ballesté Isern \\ Universitat Pompeu Fabra \\ eduard.balleste@upf.edu
}

Recepción: 27-07-2018

Aceptación: 22-11-2020

Publicación: 25-02-2021

\section{Resumen}

En el presente trabajo se analiza el activismo juvenil en el espacio de la Plataforma de Afectados por la Hipoteca de Lleida entre los años 2014 y 2017. Por un lado, se busca comprender cómo modifica al propio movimiento el incremento de la participación juvenil, observando cómo las dinámicas de movilización que surgen del 15M son impugnadas en la PAH a través de los jóvenes estudiados que promulgan un incremento de las acciones y un aumento de la acción directa como forma de protesta. Con ello, buscan resignificar las formas de protesta hechas hegemónicas a través del $15 \mathrm{M}$ y recuperar otras prácticas políticas. Por otro lado, los cambios internos que se viven en la PAH muestran la presencia de relaciones de poder y de grupos enfrentados en el seno del movimiento que van cambiando en función de las diferentes acumulaciones de capital militante. Por último, el movimiento, como consecuencia del incremento de la participación juvenil y del cambio de las dinámicas de movilización, pasa a ocupar un lugar de menor centralidad dentro del espacio de los movimientos sociales de la ciudad.

Palabras clave: participación política; activismo juvenil; capital militante; identificaciones; poder; juventud; movimientos sociales 
Abstract. The new politics: power distributions, activism and leaderships in the PAH. A case study on youth activism in Lleida.

In the present work the youth activism in the place of the Platform of Affected by the Mortgage of Lleida between the years 2014 and 2017 is analysed. It seeks to understand, on the one hand, how the increase in youth participation modifies the movement itself. Observe how the mobilization dynamics that emerge from the $15 \mathrm{M}$ are contested in the PAH through the young people studied who promote an increase in actions and an increase in direct action as a form of protest. With this, they seek to (re)signify the forms of protest made hegemonic through the $15 \mathrm{M}$ and recover other political practices. On the other hand, the internal changes that are taking place in the PAH show the presence of power relations and groups in conflict within the movement, which change depending on the different accumulations of militant capital. Finally, the movement, as a consequence of the increase in youth participation and the change in the mobilization dynamics, comes to occupy a less central place within the space of the city's social movements.

Keywords: political participation; youth activism; militant capital; identifications; power; youth; social movements

\section{Sumario}

1. Introducción 6. De grupos militantes, identificaciones

2. Aproximación teórica y metodológica

4. Capital militante y poder en la asamblea y posiciones políticas

7. La PAH: de central a periférica

Financiación

5. Hegemonías dentro de la lucha

Referencias bibliográficas

\section{Introducción}

El presente trabajo pretende ahondar en el análisis del activismo político juvenil en los espacios de movilización política surgidos o potenciados a partir del movimiento $15 \mathrm{M}$ de 2011. Para poder observar las consecuencias que dejó dicho movimiento, se analizará el papel que desempeñan los jóvenes en los movimientos sociales actuales, ya que se incluyeron entre los principales protagonistas de la movilización indignada (Feixa y Nofre, 2013; Morán y Benedicto, 2016) y siguen siendo centrales en el actual panorama político «no institucional».

El análisis se ha centrado en la participación de jóvenes activistas de 20 a 30 años en la Plataforma de Afectados por la Hipoteca de Lleida (PAH) ${ }^{1}$ entre los años 2014 y 2017. En ese sentido, se busca observar cómo influye en el

1. La Plataforma de Afectados por la Hipoteca (PAH) se constituyó antes del 15M, en 2009, pero fue tras dicho movimiento cuando se expandió por todo el Estado y creció exponencialmente. Su principal objetivo, de forma resumida, es la lucha contra los desahucios hipotecarios y las deudas de los afectados con los bancos. Para más información sobre la plataforma, entre otros, véase Colau y Alemany (2013). 
propio movimiento y en sus dinámicas la aparición de jóvenes activistas que participaron en el $15 \mathrm{M}$.

A través del análisis sobre los cambios que produce la participación juvenil en un movimiento como la PAH de Lleida, se podrán observar y analizar las concepciones diferenciadas que tienen los agentes sobre las prácticas, los discursos y las acciones que debe llevar a cabo el movimiento. Así, se busca comprender qué relaciones se establecen entre grupos dentro del movimiento y observar cómo estas grupalidades diferenciadas (Barth, 1966; Tilly, 2005) producen luchas de poder, identificaciones y relaciones desiguales (Brubaker y Cooper, 2000) que modifican el proyecto político del movimiento y las formas de acción establecidas.

Para ello, el trabajo parte de una concepción de la política en sentido amplio, que supera los límites de la institucionalidad aunque tiene en cuenta la relación con ella (Vommaro, 2015), y donde se engloban las acciones que se llevan a cabo, los significados que producen los participantes a cada acción, los discursos que se establecen y también las relaciones entre distintos activistas y grupos de activistas en función de las afinidades o de los proyectos (Yates, 2015). En ese sentido, los movimientos sociales, lejos de ser un espacio de completa horizontalidad y libres de relaciones de poder, aquí se entienden como espacios de conflictos y disputas, que normalmente se centran en los significados que los distintos participantes atribuyen a la política y a las formas de reivindicación (Pleyers, 2011). Este estudio de caso permitirá arrojar luz a las formas de activismo juvenil que emergieron del 15M (o se vieron influidas por este), así como a las relaciones que estos activismos juveniles mantienen con los movimientos sociales existentes en la ciudad. Finalmente, permitirá comprender qué consecuencias tuvo el $15 \mathrm{M}$ en los recorridos políticos de los jóvenes estudiados y observar, a través de las relaciones entre distintos grupos dentro del movimiento, qué implicaciones tiene la variable de la edad (siempre, como veremos, en conjugación con otras intersecciones) en la evolución de la PAH de Lleida.

Por último, todo ello permitirá constatar las diferentes relaciones de poder y la construcción de hegemonías que se producen en el interior de los movimientos sociales. Es decir, cómo, en función de las relaciones internas entre grupos de activistas, el movimiento toma un camino político u otro. Ello ayuda a entender la posición del movimiento dentro de las luchas sociales de la ciudad, así como la producción de invisibilizaciones y criminalizaciones que algunas veces recaen en los jóvenes activistas politizados dentro del espacio de los movimientos sociales (Mathieu, 2019) y del espacio social general (Bourdieu, 1989).

\section{Aproximación teórica y metodológica}

El trabajo se divide en cuatro partes analíticas. Primero, se exponen las distintas trayectorias que vivieron los activistas vinculados al $15 \mathrm{M}$. Esto permitirá ubicar el objeto de estudio y entender quiénes son y qué tipo de experiencias mili- 
tantes tienen los jóvenes que se involucran en la PAH. Segundo, se analizarán las distintas relaciones de poder que existen dentro de la PAH en diferentes momentos históricos a través del concepto de capital militante (Matonti y Poupeau, 2004). Ese capital se establecería a través de una acumulación diferenciada de capitales simbólicos, escolares y políticos (Mauger, 2003; Razquin, 2016), y permitirá observar tanto las relaciones desiguales dentro del grupo como la posición que ocupa cada agente. Tercero, dependiendo de las distintas acumulaciones de capital por parte de los activistas participantes, se observan diferentes estrategias políticas en el movimiento, lo que permite hablar de diversos procesos de construcción de hegemonías (Gramsci, 2013) que marcan en cada momento unas formas de acción, de organización y unos discursos específicos. Cuarto, el análisis sobre las posiciones diferenciadas (a través de la idea de capitales) y las estrategias seguidas por el movimiento (hegemonías) visibilizarán un cambio en las formas de acción de la PAH, que, a su vez, tiene efectos en la posición que ocupa el movimiento dentro del espacio político y social de la ciudad. Este último punto se centrará en comprender las consecuencias que tuvo el cambio interno de la PAH en la relación con otros agentes políticos y sociales de la ciudad a través de la creación de grupos de activistas diferenciados.

El acercamiento metodológico que se propone para la comprensión del activismo político juvenil es planteado a través de la expansión de técnicas etnográficas. Al mismo tiempo, se basa en una aproximación al entendimiento del activismo político juvenil de Lleida a través de la realización de 30 entrevistas a activistas con posicionamientos políticos diferenciados - todos ellos participantes de forma activa en el $15 \mathrm{M}$-, que permiten ahondar en la construcción de las propias trayectorias políticas (especialmente, las de los jóvenes analizados), el análisis de sus discursos y prácticas, y la visibilización de sus redes relacionales e identificaciones dentro de la $\mathrm{PAH}$.

Las entrevistas realizadas conjugan tanto agentes que participaron en el $15 \mathrm{M}$ como personas actualmente vinculadas a los movimientos post-15M. De los 30 entrevistados, 20 tienen entre 20 y 30 años, y la mayoría de ellos en el momento de la investigación participaban de forma activa tanto en algún movimiento post- $15 \mathrm{M}$ como también en colectivos autodenominados anticapitalistas, integrados por grupos anarquistas, comunistas o de la izquierda independentista. Las entrevistas se han acompañado de una observación participante prolongada a lo largo de más de dos años con la PAH de Lleida que ha permitido tanto la construcción histórica del movimiento como el análisis de su evolución. ${ }^{2}$

2. El trabajo etnográfico en el marco de la tesis doctoral (Ballesté, 2018) tuvo lugar entre 2014 y 2017, y más allá de las 30 entrevistas contó con la construcción de cuatro trayectorias políticas de jóvenes, la observación participante en movimientos y colectivos durante dos años (principalmente, pero no únicamente, la Marea Blanca y la PAH), dos grupos de discusión, una revisión de los documentos de los movimientos y de noticias de prensa y, finalmente, la aplicación de metodologías para aprehender la construcción de redes sociales entre los distintos actores (Molina, 2001). 
La posición que se toma en el acercamiento al campo de estudio es una de las disyuntivas más importantes del trabajo. Desarrollar un proceso etnográfico en una ciudad conocida (Hammersley y Atkinson, 1994), analizar espacios y actores, algunos de los cuales ya eran conocidos por el investigador, e intentar comprender una realidad cercana implica que el proceso reflexivo que acompaña todo el estudio aumente considerablemente (ibíd.; Ferrándiz, 2011).

El debate, a su vez, se sitúa en medio de lo que se ha denominado la etnografía implicada, políticamente comprometida o militante (Scheper-Hughes, 1995; Nash, 2008; Graeber, 2009; Juris, 2007; Juris y Kashnabish, 2013), es decir, en la disyuntiva sobre el papel que debe tener el investigador al estudiar luchas sociales o emancipadoras. En esa línea, parece muy adecuada la explicación que aporta Nash de los nuevos retos o paradigmas de la antropología de los movimientos sociales. La autora reflexiona sobre la postura del antropólogo en su relación con el campo estudiado de luchas sociales y de grupos oprimidos. Bajo el nombre de "paradigma para el sistema global emergente», muestra cuatro temas centrales o problemáticas que debe enfrentar el investigador, sobre los cuales dicho trabajo se ha apoyado y servido para la conformación de todo el proceso etnográfico: 1) Participación activa en el contexto social del grupo que se está estudiando, 2) autorreflexión en la interacción con el otro y autocrítica de todas las interpretaciones en el campo de estudio, 3) atención a la realidad material, y 4) creación de espacios para el intercambio cultural. (Nash, 2008: 19)

A su vez, asumiendo que la juventud —y con más énfasis, la politizadasigue siendo uno de los sectores poblacionales más estigmatizados en este sistema cultural y sociopolítico neoliberal (Claret, 2013), es necesario analizar las distintas violencias que se les aplican (o adjudican), y también ver cómo reaccionan frente a ellas dichos jóvenes politizados (Valenzuela, 2015b). A menudo, muchos de los colectivos que atraviesan constantemente la etnografía suelen recibir, por parte de las instituciones y de los medios de comunicación (entre otros), campañas de categorización/etiquetación que los describen como violentos, radicales y antisistema (Feixa et al., 2014). En este análisis se busca, como ejercicio paralelo, profundizar en estas cuestiones y arrojar luz a estas categorías que reducen y estigmatizan la realidad política juvenil.

Por último, entre los distintos movimientos sociales de la ciudad, se escoge la PAH como principal espacio de participación juvenil e interrelación con lo aquí llamado post-15M. Otros movimientos, como las mareas a favor de los servicios públicos o espacios propiamente juveniles, no permitieron observar esta interrelación entre agentes (jóvenes y adultos), ni ver de forma clara las relaciones internas diferenciadas. A su vez, como reconocen varios de los agentes entrevistados, de todos los movimientos que emergieron (o se reconfiguraron) después del $15 \mathrm{M}$, este es el que producía una mayor atracción sobre los jóvenes politizados, tanto por su «motivo» de lucha como por el perfil de la gente participante. Finalmente, la elección de un movimiento en una ciudad como Lleida, aun no ser representativo de lo sucedido en otras ciudades (sobre todo de tamaños diferentes), permite obtener un conocimiento más profundo 
de los efectos del 15M en una ciudad mediana que, en última instancia, puede encontrar vínculos comparativos con otros estudios de caso específicos.

\section{Movilización juvenil post-15M $\mathrm{M}^{3}$}

La "generación indignada» (Feixa y Nofre, 2013), que llenó las principales plazas del país en el año 2011 y marcó un punto de ruptura en la concepción que se pretendía tener de la juventud como agente despolitizado y desinteresado -ninis - (Navarrete, 2011; Strecker et al., 2018), fue uno de los principales acontecimientos políticos recientes de movilización (Byrne, 2013; Feixa et al., 2015). Pasados unos años de la expresión de la indignación, la situación laboral, económica y sociopolítica con la que se encuentra a día de hoy la juventud no ha cambiado en gran medida (Cabasés et al., 2018). Este modelo en crisis sigue atacando a los sectores más jóvenes de la sociedad y lleva consigo lo que se ha llamado un juvenicidio moral: proceso que surge del sumatorio del juvenicidio económico y del simbólico (Strecker et al., 2018) — trasladando el concepto aplicado por José Manuel Valenzuela para el caso mexicano (2012) — y que consiste en:

...el proceso social de «exterminio» moral de la juventud, como período vital y como condición social, en el sur de Europa, en el período posterior a la crisis financiera de 2008, y sobre sus efectos en el mercado laboral y en los imaginarios sobre la juventud. (Feixa et al., 2015: 237)

Ante este escenario permanente de no futuro, y siendo los jóvenes unos de los principales damnificados de las políticas aplicadas de austeridad, se presenta de mayor relevancia el análisis de la situación política juvenil (Valenzuela, 2015a). Analizar el contexto actual del activismo político juvenil permite acercarnos al conocimiento de cómo están reaccionando estos jóvenes politizados a una situación de crisis - moral y económica — que se enquista y parece no revertir. Para hacerlo, se centra el trabajo cualitativo en la información recogida en la ciudad de Lleida (Cataluña), un estudio local que permite analizar de forma profunda el formato de un movimiento de carácter estatal (la PAH) que, en distintos formatos y magnitudes, está presente en la mayoría de ciudades del país. Por otro lado, dicho enfoque "micropolítico" (Scott, 1985; Gledhill, 2000), que busca analizar las relaciones que se establecen dentro de un movimiento en su día a día, permite comprender las consecuencias del propio movimiento $15 \mathrm{M}$ y analizar cómo modificó las formas políticas contestatarias existentes anteriormente en la ciudad.

A través de las distintas entrevistas realizadas a participantes del $15 \mathrm{M}$ en la ciudad, se ha podido vislumbrar que, en la mayoría de los casos, estos agentes

3. Utilizamos el concepto post-15M introducido por José Mansilla en «Movimientos sociales y apropiaciones colectivas en la Barcelona post-15M: el papel de la Assemblea Social del Poblenou» (2015). 
se encontraban en el post-15M en una de estas tres situaciones: 1) de menor intensidad de participación y en un proceso, por diferentes motivos, de cierta desmovilización; 2) de trasvase del activismo hacía otros espacios políticos institucionales; ${ }^{4}$ 3) de incremento de la politización y participación en espacios políticamente más activos o movilizados. Para categorizar dicha evolución, se sigue la descripción que llevan a cabo Porta y Diani (2011) de los «caminos posibles» de los movimientos sociales —institucionalización, radicalización, comercialización, desactivación, entre otros-, con el traspaso de algunos de ellos a los trayectos que siguen los activistas aquí estudiados. Estos tres "caminos posibles» en la evolución de los participantes del $15 \mathrm{M}$ permiten describir de forma general los perfiles y las características de aquellos que se integraron en la PAH.

\subsection{Desmovilizados}

El post-15M se caracterizó por abrir un proceso de sectorialización y territorialización del movimiento de los indignados que dio lugar a la creación o potenciación de movimientos sociales centrados en luchas más específicas o locales (las mareas por los servicios públicos, la $\mathrm{PAH}$ o las representaciones del movimiento en distintos barrios son un ejemplo de ello) (Pastor, 2013; Mansilla, 2015). En Lleida, todos esos espacios tuvieron una menor participación juvenil, y su creación coincidió con un proceso de cierta desactivación política de algunos de los jóvenes indignados (Ballesté, 2018).

Los caminos políticos de los jóvenes que habían ocupado la plaza de la ciudad fueron varios. Algunos de ellos durante el $15 \mathrm{M}$ se encontraban en la etapa universitaria, aunque vinieran de otras ciudades tanto catalanas como españolas. Eso produjo que, una vez terminados sus estudios, abandonasen la ciudad. Como ejemplo, uno de los grupos más numerosos de estudiantes del $15 \mathrm{M}$ estaba formado por alumnos de la Facultad de Medicina. En Lleida, se trata de la titulación universitaria que acoge más estudiantes de fuera de la ciudad. Empujados por los recortes implantados en sanidad, estos universitarios se unieron al nacimiento de dicho movimiento y, una vez terminada su etapa de formación, volvieron a sus ciudades natales o se movilizaron laboral $\mathrm{o}$ académicamente en otras partes del territorio.

Otro de los factores era la reducción de la participación juvenil en los movimientos post-15M y una implicación mucho más puntual en manifestaciones o acciones más masivas. Esto va relacionado con un cambio de las reivindica-

4. Es necesario destacar que en ese proceso de inicio etnográfico se produjeron hasta cuatro citas electorales distintas (unas municipales, unas autonómicas y dos estatales), lo que afectó de forma directa a todo el campo activista de la ciudad. Así, aunque a distintos niveles e intensidades, todo el nuevo engranaje electoral que acompañó dichas citas (sobre todo a partir de la aparición de nuevos partidos) conllevó una acumulación de capital humano hacía distintos partidos políticos, en muchos casos proveniente de los movimientos sociales. Para un detallado análisis de la política aquí llamada institucional en el post-15M, véase Tugas (2014). 
ciones políticas de estos movimientos más sectoriales que, centrados muchos de ellos en cuestiones como la sanidad o la educación, significaban una interpelación menos directa con las problemáticas juveniles. Jóvenes como Gabriel, ${ }^{5}$ uno de los más implicados en el proceso de las acampadas, buscaron nuevos espacios de lucha política más autónomos centrados en proyectos concretos:

Ahora mismo estoy en..., estamos intentando reactivar la red ecológica de Lleida (...), un poco por la soberanía alimentaria, un poco por esta línea de crear una red de intercambio de productores y consumidores y utilizar (una) moneda social y apostar por el cambio social de una forma más integral.

Para él, el activismo político se había convertido también en una lucha personal de su día a día, en promulgar la autogestión y la implicación consigo mismo. Aun así, como seguía contando, se sentía con una ligazón con los movimientos donde había participado y asistía de forma eventual a las acciones más masivas. En este caso, el desgaste producido por la movilización constante y la necesidad de empezar la revolución por uno mismo (Pleyers, 2013) habían provocado una disminución del grado de asistencia en los movimientos sociales presentes. En esa línea, siguiendo a Giugni et al. (2016), se puede observar que, aunque muchos de estos agentes hayan reducido su participación, el propio $15 \mathrm{M}$ impactó de forma directa en sus vidas y tuvo unas consecuencias duraderas y posteriores (desde el apoyo puntual a manifestaciones hasta, por ejemplo, el cambio en hábitos personales).

Por su parte, Xavier ${ }^{6}$ explicaba que, después de verse envuelto en un proceso judicial, relajó su implicación en el activismo y pasó a dedicarse a proyectos personales para evitar tener problemas jurídicos. Aun así, sigue considerando que el activismo, o la lucha política, es una parte muy importante de su vida personal, pero, como expresa, «hay que escoger un camino y centrarse en lo que uno quiere».

De forma general, los entrevistados coinciden en destacar tanto la disminución de la movilización juvenil en una ciudad como Lleida después del 15M, como la articulación de nuevos proyectos políticos más específicos o expresamente juveniles. Aunque para la mayoría el 15M significó un cambio sustancial en sus trayectorias políticas, en el sentido de apostar por volver a movilizarse

5. Entre paréntesis, incorporaciones del autor. Todos los nombres aquí expuestos han sido cambiados y no son los verdaderos. Entrevista realizada el 4 de marzo de 2015. Hombre de 26 años en el momento de la entrevista. Estudiante universitario. Estuvo implicado en distintos movimientos universitarios. Durante el $15 \mathrm{M}$, fue un agente muy activo. A través de este movimiento, se implicó en la creación de distintos grupos de indignados barriales y sectoriales, en los que destaca la Coordinadora de Mareas. En el momento de la etnografía, aunque había bajado su nivel de implicación, seguía participando en actos de la PAH, la Marea Blanca y otros movimientos de la ciudad.

6. Entrevista realizada el 18 de abril de 2015. Informático e ilustrador gráfico. Hombre de 27 años en el momento de la entrevista. Aunque había participado en movimientos sociales anteriores, fue el 15M el espacio de mayor integración. Se implicó en las acampadas, en la organización, en los debates, etcétera. Posteriormente, también participó en la creación de las mareas. 
en cualquier momento, no lo hacen de forma constante en movimientos como las mareas o la PAH.

\subsection{Institucionalizados}

Por otro lado, el año 2015 vino marcado por un calendario con distintas citas electorales. Este hecho provocó que la política institucional se situase en el centro del panorama político de la ciudad e influyó al resto de espacios políticos existentes. Algunos de esos nuevos proyectos institucionales apelaban directamente a ideas que emanaron del $15 \mathrm{M}$, haciendo que algunos partidos se autoidentificaron como los herederos del propio movimiento (Castells, 2015; Subirats, 2015).

En ese sentido, Bruno ${ }^{7}$ explicaba que se encontraba justo en un proceso de apuesta por la política institucional al presentarse como cabeza de lista por un partido de «izquierdas tradicionales». Este hecho le provocó un distanciamiento de los movimientos sociales donde había participado con anterioridad y donde había desempeñado un papel principal (la PAH fundamentalmente). Una desconexión que se explicaba, según él, por el desgaste vivido durante años en el movimiento, por motivos éticos y por no mezclar o apropiarse de la fuerza de dicho movimiento en la lucha electoral que empezaba. Como podemos ver, en los movimientos post- $15 \mathrm{M}$ hubo activistas que a su vez participaban en proyectos políticos institucionales lo que, algunas veces producía conflictos internos que, como en el caso de Bruno, terminaban por una mayor implicación en los partidos y un cierto abandono de los movimientos.

Al mismo tiempo, la observación participante desarrollada permitió ver una dispersión de personas que se habían implicado en el 15M, normalmente de edad más avanzada (mayores de 30 años en su mayoría), hacía grupos políticos de izquierdas, tanto existentes como emergentes.

Por tanto, la aparición de una brecha de oportunidad política o de un espacio electoralista que procura aunar los reclamos del movimiento $15 \mathrm{M}$, pero con un formato de lucha institucional, provocó ya no solo la desmovilización de algunos agentes, sino también el traspaso de sus fuerzas a la lucha electoral a distintos niveles. En este sentido, Tilly y Wood (2010) explicitan distintos escenarios posibles para los movimientos sociales, entre los cuales estarían la profesionalización de la protesta y por tanto su institucionalización. Para este caso, no se hace referencia a la institucionalización del propio movimiento, ya que el 15M como tal en Lleida no desembocó de forma unívoca en un partido político, pero sí a un trasvase de actores del movimiento hacía plataformas políticas electorales, que en algunos casos es puntual — vía cita electoral—y en otros permanente.

7. Entrevista realizada el 23 de diciembre de 2014. Hombre de 37 años en el momento de la entrevista. Militante de diferentes partidos políticos. Participante activo de movimientos como el No a la Guerra, muy activo en el mismo 15M en Lleida y también en la creación de la PAH, donde fue portavoz en los primeros años. 


\subsection{Politizados}

Por último, como punto principal en dicho trabajo, con el término politizados se hace referencia a aquellos activistas del $15 \mathrm{M}$ que, una vez terminado este movimiento, siguieron participando de forma activa en otros colectivos políticos o grupos existentes o de nueva creación. Específicamente, tomando los ejemplos de la ciudad de Lleida, se hace referencia a colectivos comunistas, anarquistas, libertarios y a distintos grupos políticos de la izquierda - y de la izquierda independentista- que normalmente tienen un perfil ampliamente juvenil. ${ }^{8}$

Estos colectivos, que tuvieron una relación desigual con el $15 \mathrm{M}$ y los movimientos posteriores (Nofre et al., 2015), permiten analizar los distintos momentos de convergencia/divergencia que se crean con los espacios políticos post-15M (Feixa et al., 2014). De esa relación desigual también surgen las luchas de poder internas y las identificaciones entre grupos y movimientos que permiten entender las diferentes relaciones que tienen los jóvenes activistas con movimientos como la PAH, entre otros, a través de la valoración que hacen del propio movimiento indignado.

Así se expresa Dani, ${ }^{9}$ un joven que se activó políticamente con el 15M, al ser preguntado por su valoración sobre el movimiento: «...en parte está bien que haya movimiento, ¿no? Que la gente se mueva... Pero creo que diluyó mucho toda esa rabia que se podría haber aprovechado mucho de otra manera. Personalmente, claro, a mí me dio la vida...». Una vez finalizada la acampada en la plaza, también simpatizó con movimientos anarquistas, donde desarrolló una doble visión del $15 \mathrm{M}$ perfectamente sintetizada en la cita. Por un lado, la ilusión porque se diera dicha movilización y la entrada personal en el mundo del activismo. Por otro lado, la sensación de que, para él, el movimiento pudo canalizar y calmar mucha rabia que había entre la ciudadanía.

Este último grupo tiene especial interés para el caso aquí presentado, el que se relaciona con la evolución reciente de la $\mathrm{PAH}$ en Lleida. Prácticamente al mismo tiempo en que se desarrolló la etnografía, empezó también el crecimiento de la participación de dichos jóvenes en la plataforma, lo que produjo nuevas dinámicas y relaciones tanto dentro como fuera del movimiento y propició la

8. Todos estos colectivos han sido presentes históricamente en la ciudad. La mayoría de ellos atraen a buena parte de la juventud politizada y movilizada, y se componen principalmente de jóvenes entre 16 y 30 años. Los colectivos citados han tenido fases de crecimiento y decrecimiento desigual, pero siempre se han mantenido (cambiando a veces de forma) en la vanguardia de la lucha política no institucional. En el espacio temporal de la investigación, aparte de encontrarse en un momento de crecimiento, dichos colectivos también estaban tejiendo redes de alianzas entre ellos a través de distintas plataformas, como la Coordinadora Antifascista de Lleida, el Correscales, el 1 de Mayo Anticapitalista, entre otras.

9. Entrevista realizada el 9 de noviembre de 2016. Hombre de 26 años en el momento de la entrevista. Graduado en la universidad. Residente en Lleida desde hace años, el 15M fue su momento de activación política personal. Ha participado en algunas de sus formas posteriores como las mareas o la PAH. A través de los indignados, se implicó en movimientos libertarios y anarquistas de la ciudad. 
aparición de distintos conflictos o reconfiguraciones internas, como se verá a continuación.

En definitiva, la comprensión de los distintos caminos escogidos por los activistas del $15 \mathrm{M}$ permite centrar el grupo estudiado (los jóvenes politizados) y ponerlo dentro del contexto más general de traspase del $15 \mathrm{M}$ al post-15M. Como se ha visto, este trasvase tuvo un alto grado de pérdida de participantes y un cierto cambio de perfil en los protagonistas de los nuevos movimientos (edad más avanzada, vinculación laboral con distintos servicios públicos, situación de vulnerabilidad por pérdida de viviendas, etcétera). En este contexto, las críticas que emergen por parte de los jóvenes politizados al propio $15 \mathrm{M}$ serán un elemento central para comprender las luchas de poder y los conflictos que se reprodujeron en la PAH a través de la participación juvenil politizada.

A continuación, por jóvenes politizados se entenderán aquellos que mayoritariamente participaron en el $15 \mathrm{M}$ y que posteriormente, una vez finalizadas las acampadas indignadas, pasaron a formar parte de colectivos políticos próximos al anarquismo, al comunismo o a la izquierda independentista. Aunque se trata de una categoría generalizante, permitirá englobar dentro de un grupo no homogéneo jóvenes que coinciden habitualmente en una apuesta por la acción directa como herramienta política, en el asamblearismo como forma de organización y que, a su vez, se agrupan de forma puntual en campañas unitarias de carácter anticapitalista (como los mismos grupos exponen).

\section{Capital militante y poder en la asamblea}

Entender el contexto y la evolución que vivió el movimiento $15 \mathrm{M}$ a través de los caminos que escogieron sus activistas permite empezar a construir ciertas trayectorias políticas e identificar qué perfiles de jóvenes, en nuestro caso, siguieron participando y de qué forma en los movimientos post-15M. Para ello, a continuación, se describe y analiza cómo fue el paso de estos jóvenes politizados del $15 \mathrm{M}$ a la $\mathrm{PAH}$ de Lleida.

La PAH de Lleida, a mediados de 2013, era uno de los movimientos más multitudinarios de la ciudad. En aquella época se reunía en los bajos de una iglesia (Santa María Madalena) cedidos por el propio obispado. La asamblea estaba compuesta por unas 60 personas, y había una clara distinción entre dos perfiles: los afectados y los activistas. ${ }^{10}$ Dentro, un grupo de tres personas llevaban la asamblea, entre los cuales había un portavoz principal que habitualmente ejercía de punto de referencia para el resto de participantes. La función de dicho portavoz era la de centralizar la mayoría de las cosas que sucedían en el movimiento y, a su vez, hacer de cara visible del movimiento hacía el exterior.

En ese momento, después de un primer periodo (justamente después del $15 \mathrm{M})$ marcado por una avalancha de atención de casos de desahucios y la falta

10. Los propios participantes de la PAH de Lleida establecen esta diferenciación de perfiles. Los afectados serían aquellos que acuden a la plataforma con una problemática de vivienda o hipotecaria. En cambio, los activistas serían aquellos que participan en la $\mathrm{PAH}$ de forma solidaria. 
de medios y conocimiento para atenderlos o pararlos, el movimiento empezó a establecer su configuración interna y su forma política de actuar. Esto lo convirtió, poco a poco, en un movimiento de atención y resolución de problemáticas ligadas a la vivienda. En esos primeros años, el movimiento se erigió en un espacio funcional para hacer frente a las necesidades inmediatas y urgentes de una parte de la población afectada que acudía a él. ${ }^{11}$ Desde ese inicio, dicho portavoz ejercía, sin explicitarlo claramente, un alto grado de liderazgo dentro del movimiento que se visibilizaba en sus intervenciones en las asambleas, en la materialización de qué acciones debían abordarse —o qué orientación debían tomar- y en la ayuda personal a los afectados. Así, este portavoz iba acumulando un alto grado de capital simbólico (Bourdieu, 1986) o militante (Matonti y Poupeau, 2004, que le permitía situarse como punto de referencia para el movimiento a partir de la acumulación de experiencias y saberes (tanto en su militancia anterior en el propio $15 \mathrm{M}$ como en esos primeros compases de la PAH de Lleida).

Los nuevos asistentes, la mayoría afectados por casos hipotecarios con una situación a menudo precaria, lo posicionaban como punto de apoyo principal y referente. Su facilidad para hablar en público, su «autoridad carismática» (Weber, 2007) representada a la hora de construir discursos, los conocimientos previos en estas y otras materias, y una implicación temporal muy elevada lo situaban como líder o referente indiscutible (Bourdieu, 1989).

Este formato de la $\mathrm{PAH}$, como espacio político centralizado bajo el liderazgo del portavoz, provocó que distintos agentes que habían participado en el 15M (especialmente los aquí llamados jóvenes politizados) no se sintieran representados por el formato del movimiento, ni tampoco por las prácticas y los discursos de su líder. Uno de los problemas más señalados era la distancia política con la visión de la lucha que tenía ese portavoz y que, por extrapolación, seguía todo el movimiento. Como explica Pedro, ${ }^{12}$ su proceso de acercamiento y distanciamiento con el movimiento vino marcado por esto: «... y entré a la PAH (...) hace 4 años y lo dejé porque estaba el portavoz. (...) Y lo que veía era superior a lo que podía aguantar y, no lo sé, proponía cosas y siempre... No lo sé, controlaban todo el cotarro».

A partir del año 2014 toda la situación cambió. El hasta entonces portavoz abandonó su posición y al propio movimiento, lo que, después de un breve periodo con cambios de portavoces, abrió un nuevo espacio interno de debate sobre cómo debía organizarse la PAH. Así, después de momentos de incertidumbre vividos por los nombramientos de distintos portavoces — siempre afines al antiguo portavoz-, y con la intermediación de la organización

11. La evolución de la PAH de Lleida se reconstruye a partir de la propia experiencia personal junto con una entrevista realizada a su portavoz a través del proyecto GENIND (del 10 de octubre de 2013) y de distintas entrevistas posteriores.

12. Entrevista realizada el 9 de mayo de 2015 . Hombre de 28 años en el momento de la entrevista. Miembro de un colectivo comunista, participó activamente en el $15 \mathrm{M}$ y también en las distintas ramificaciones posteriores como las mareas u otras plataformas. Su mayor implicación se dio en la PAH. 
catalana de las PAH (que dispone de un grupo de mediación para problemáticas diversas), el movimiento decidió dejar de tener portavoces y potenciar el modelo asambleario de autorrepresentación propia de cada actor participante. Con ello, se produjo un vacío de poder, o una desaparición del liderazgo, que permitió la participación y la adopción de nuevos roles a los activistas ya presentes y abrió un espacio de oportunidad para la participación de los jóvenes politizados anticapitalistas.

La nueva fase se basó en la idea de que cada persona afectada se ocupaba de su propio caso en un proceso de igual responsabilidad, y finalmente en la asamblea recaía el poder de tomar todas las decisiones conjuntas, como por ejemplo las acciones o las campañas a realizar. Así, se potenciaba el modelo asambleario de toma de decisiones debatidas, consensuadas y democráticas (Graeber, 2011). Al mismo tiempo, se abandonaba el modelo anterior, donde un grupo de personas escogidas por sus conocimientos en materia de vivienda e hipotecas estudiaban los casos, negociaban con los bancos y, en definitiva, ejercían de interlocutores del afectado. ${ }^{13}$

Involuntariamente, esta reconfiguración del propio movimiento produjo un reposicionamiento tanto de los miembros de la plataforma ya presentes como también de otros agentes que con anterioridad se habían acercado al movimiento sin sentirse suficientemente cómodos para seguir militando en él. Natalia, ${ }^{14}$ una activista de la PAH que vivió la mayor parte de dicho proceso, explica: «Una vez han pasado las municipales, las elecciones, entonces esta gente [líder y partidarios] se han retirado (...), y entonces la PAH vuelve un poco a aceptar (...) que otras voces (...) puedan hablar perfectamente y no pase nada...».

La materialización de dicho proceso también se observó dentro de la asamblea en el cambio de la distribución entre los asistentes. En el primer modelo, el público se situaba sentado en filas y los portavoces, en la tarima detrás de una mesa, presidían las asambleas. En el segundo, la tarima y las filas fueron sustituidas por diversos círculos concéntricos donde todos los asistentes se sentaban en una posición similar — más o menos alejados del centro.

Aun así, esta nueva disposición espacial también visibilizó nuevas distribuciones de poder y de liderazgo que se materializaban a través de la cercanía (o no) a las filas centrales. Las primeras líneas eran ocupadas por aquellos participantes que más intervenían en las asambleas, y las últimas eran ocupadas normalmente por los afectados que acudían por primera vez o por gente que no participaba mucho.

13. Como se pudo observar, cabe especificar que, aun habiéndose producido este cambio en el modelo organizativo, aquellas personas con una experiencia o unos conocimientos mayores tendían a ayudar a los afectados más nuevos en un proceso de empoderamiento.

14. Entrevista realizada el 24 de febrero de 2015. Entre paréntesis, incorporación del autor. Mujer de 36 años en el momento de la entrevista. Ingeniera forestal. Aunque su participación en el $15 \mathrm{M}$ no fue muy activa, posteriormente se implicó de forma profunda en la PAH. Vivió las distintas fases del movimiento y ocupó un lugar importante en cuanto a relaciones con la institución. 
La eliminación de la figura del portavoz, más allá de generar la participación de nuevos agentes en el movimiento, también produjo una reconfiguración de las relaciones de poder internas. Los activistas con una acumulación mayor de capital militante, normalmente situados en las primeras filas de los círculos, pasaron a ser los dinamizadores de las asambleas. Este cambio en las relaciones de poder permite observar que este, lejos de desaparecer en la segunda fase, se distribuyó entre otros agentes. Así, aunque el nuevo modelo se acercase más a un concepto de democracia directa y participativa sobre el que jóvenes actores politizados se sentían más representados, las relaciones de liderazgo seguían produciéndose, basadas en el concepto de capital militante citado y en la implicación, las experiencias acumuladas y la fuerza discursiva de los agentes.

En el paso de la primera a la segunda fase, se materializaron tanto las oportunidades de participación que se abrían para jóvenes politizados (propiciadas por el abandono del modelo de portavoces anterior) como las reconfiguraciones internas en el rol que cada agente tenía dentro del movimiento. Dicho proceso de reconfiguración, a su vez, pivotaba en concepciones distintas de entender las formas y la función que debía tener la $\mathrm{PAH}$, que se pueden resumir y catalogar con los conceptos de reformistas/rupturistas (Nofre et al., 2015).

\section{Hegemonías dentro de la lucha}

Como se ha introducido, en el espacio de vacío de poder que se abrió entre el paso de fases, aparecieron en la plataforma varios agentes provenientes de distintos colectivos de la ciudad (con un perfil mayoritariamente juvenil), llamados por las oportunidades que se estaban generando en la batalla por cambiar o profundizar los objetivos y las luchas de la PAH. Esto se manifestaba de forma clara en las asambleas que tenían lugar los viernes por la tarde, que se centraban en decidir el camino político del movimiento. ${ }^{15}$ Principalmente, los jóvenes que incrementaron su participación en la PAH provenían de dos colectivos políticos: uno anarquista y otro comunista. Estos colectivos, como expresaba informalmente uno de sus miembros a la salida de una asamblea de la PAH, habían visto una brecha de oportunidad para poder expresar sus ideas políticas, sus formas organizativas y sus aportaciones en materia de luchas y asamblearismo, lo cual se había transmitido de unos a otros para aumentar el volumen de participantes. Pedro, uno de dichos jóvenes que habían empezado a participar, explicaba:

A los libertarios, sobre todo los convencimos nosotros [los comunistas] de que se implicasen... y, quien dice los libertarios, ojalá también viniesen, yo que

15. La PAH se organizaba a partir de dos asambleas. Los lunes se celebraba la asamblea de bienvenida, donde se presentaban y trataban nuevos casos de afectados, y también se repasaba la situación de casos más viejos. Los viernes se convocaba una asamblea más organizativa o política, donde se definían las acciones venideras y el camino político de la organización. En cada asamblea se veía perfectamente el perfil que adoptaba cada participante. Los más preocupados por la evolución política de la PAH solían asistir a las de los viernes. 
sé, la gente de Arran (colectivo de jóvenes de izquierdas independentistas). Cuantos más seamos que queramos ir un paso más allá de lo que propone la PAH, mejor.

Después de celebrar algunas asambleas, dichos jóvenes tenían un papel protagonista en el funcionamiento del movimiento. De dos o tres que acudían a las primeras asambleas, al cabo de pocos meses ya eran alrededor de diez, y el número iba incrementando si se trataba de acciones concretas, como manifestaciones u ocupaciones de sedes bancarias. Su implicación había crecido hasta el punto de que algunos de ellos asistían como representantes a los encuentros de PAH catalanas y españolas, y participaban en todas las acciones (encierros, protestas, pegatinadas y manifestaciones), lo que les llevo a que algunos acabaran denunciados ante los tribunales por su participación en ocupaciones bancarias. Eran protagonistas y su alto grado de implicación y compromiso les otorgaba una fuerte legitimidad delante del resto de la asamblea, lo que se traducía en una acumulación elevada de capital militante. Estos cambios en los roles internos provocaron la aparición de distintas luchas por hacer hegemónico, en un sentido gramsciano de consenso, un modelo de concebir la lucha y la política, tanto en un formato práctico como discursivo, dentro del movimiento (Gramsci, 2013; Feixa, 2014).

Las concepciones políticas que seguían estos jóvenes en sus propios colectivos, con un modelo de democracia directa basada en el asamblearismo, la horizontalidad y la acción directa como formas de manifestarse (Graeber, 2011), se introdujeron en la PAH y generaron varios debates entre sus miembros. Uno de los más importantes se centró en la violencia y sobre todo en delimitar qué era violencia, cuál estaba justificada y quién ejercía más violencia - la protesta en sí o el Estado y los poderes fácticos. En esos debates se materializó la aparición de diferentes grupos dentro del movimiento que se enfrentaron a la hora de delimitar qué camino seguiría la PAH.

En este punto, es interesante poner la mirada en el $15 \mathrm{M}$ para observar las concepciones hegemónicas que dejó en temas como la violencia o la acción directa (Taibo, 2013). La censura radical que se realizó desde el movimiento de los indignados a formas de protesta que pudieran ser vistas por la opinión pública como violentas también se trasladó a la mayoría de movimientos post-15M. Ya en el $15 \mathrm{M}$, este fue uno de los principales puntos de discrepancia entre los activistas y, al menos en el caso de Lleida, produjo enfrentamientos entre activistas que participaban en colectivos políticos con anterioridad (como, entre otros, los aquí estudiados) y otros participantes indignados. Todo esto se materializó en las plazas cuando temas como el proceso independentista catalán, el posicionamiento entre monárquicos o republicanos o, simplemente, las acciones que debían producirse en las movilizaciones se esquivaban de los debates asamblearios por temor a enfrentamientos o a generar lejanía con la simpatía popular lograda. Dicha concepción de la movilización pacífica se trasladó más allá del $15 \mathrm{M}$, lo que provocó que muchos de los activistas de la $\mathrm{PAH}$ interiorizaran estos límites de la no violencia (Ballesté, 2019). 
Al mismo tiempo, y a un nivel más general, la $\mathrm{PAH}$, desde mediados de 2012, vivía en todo el Estado un proceso de redefinición política y de acción (Colau y Alemany, 2013). La avalancha de casos de desahucios de los primeros años se había visto reducida - el poder que acumulaba la PAH era creciente y la mayoría de bancos y entidades financieras preferían negociar a verse envueltos en protestas y ocupaciones frente a sus sedes. El movimiento, ante un aumento del carácter asistencial y de acompañamiento de los casos, abrió nuevas problemáticas sobre las que centrar su combate, como la lucha contra la pobreza energética. Al mismo tiempo, se encontraba inmerso en pleno debate interno sobre la incisión política de la PAH y su capacidad emancipadora hacía los distintos participantes, activistas y afectados. Es decir, el movimiento se planteaba problemáticamente la capacidad que tenía (o no) de mantener a los afectados por casos de desahucios o de impagos de las hipotecas políticamente comprometidos, una vez resuelto su caso hipotecario particular.

En ese sentido, todos estos debates también se hacían visibles dentro del movimiento en Lleida, y coincidieron mayoritariamente con el cambio de modelo del movimiento en la ciudad. La separación principal entre posturas y grupos estaba relacionada con el debate sobre si seguir manteniendo un formato asistencial y de ayuda individualizada a los afectados o, por el contrario, buscar luchas comunes entre grupos de afectados que permitieran devolver el carácter movilizatorio del grupo y apostar por soluciones que pasasen por un cambio de modelo de vivienda general.

Para Bruno, el primer portavoz del movimiento en Lleida, existían dos tipos de asambleas de la PAH en el ámbito catalán: las cerradas o las abiertas, según su nivel de continuación de movilización o reclamos políticos. Estos modelos generales, aplicables a los grupos en cada ciudad, también se reproducían dentro del grupo en Lleida:

(...) que pasa con esto, que dentro de la PAH también hay gente, y aquí en Lleida pasa, que cree que tiene que seguir con las movilizaciones, pero claro... si ya estás negociando [con el banco]... Movilizarte porque sí, si ya estás en un proceso negociador, es difícil. Y se crea un conflicto entre los que dicen que se tiene que negociar, y seguir negociando si la negociación es fructífera, y los que ven lenta su negociación y dicen: ¡Volvamos a okupar oficinas!

En este marco, los jóvenes politizados apostaban por la lucha continuada de la PAH («ir un paso más allá» de lo que venía proponiendo el movimiento) y la búsqueda de un cambio del sistema que no solo dé solución a los distintos casos denunciados, sino que transforme el modelo hipotecario, bancario y de vivienda. De alguna forma, buscaban exportar las reivindicaciones o luchas que mantienen en sus propios colectivos a la propia PAH. ${ }^{16}$ Todo este proceso llevó a la aparición de distintos momentos de tensión interna en la PAH,

16. En cierto sentido, se pueden observar paralelismos entre estas posturas y lo que Escobar llama «alternativas a la modernidad» o "posibilidades culturales» alternativas al modelo neoliberal (Escobar, 1995; Gledhill, 2000). 
propulsados habitualmente a partir del debate constante de hasta dónde deben llegar las acciones, qué es violencia, qué están dispuestos a «sufrir» judicial y penalmente, y cuál es la finalidad de cada acción.

Los perfiles de cada uno de los grupos, que anteriormente se han definido en general como reformistas y rupturistas, permiten comprender el impacto y el seguimiento que tuvo cada posición. Así, mientras que los impulsores del incremento de la acción directa y de la idea de realizar protestas políticas que impugnen el modelo de vivienda eran mayoritariamente juveniles, con un elevado capital militante (a través tanto de la experiencia acumulada en su militancia en otros colectivos como de unos conocimientos políticos y de acción elevados) y con una cierta oposición a las formas políticas del 15M (aunque la mayoría participaron en él), los que apostaban por seguir con un modelo asistencial solían ser agentes más adultos, con un tiempo más prolongado de participación en la PAH (habían coincidido con la primera fase de portavoces), y la mayoría tenían ya resueltos sus casos personales. En medio de los dos grupos se encontraban buena parte de los «afectados», que apostaban por un cambio de modelo y un incremento de la lucha común, pero que, a su vez, veían con dificultad poder hacer frente a la represión que ese aumento de las acciones podía comportar (tanto en formato de juicios como de multas, etcétera). Este último grupo estaba conformado, mayoritariamente, por familias de clase obrera, muchas de ellas racializadas, que se encontraban en una situación de elevada precariedad.

En esta fase de reconfiguración, el movimiento acumuló distintos juicios por ocupación de entidades bancarias como consecuencia del aumento de las acciones y de su contundencia. Esto provocó un fuerte debate interno sobre si seguir con la movilización o, por el contrario, relajar temporalmente las acciones hasta calmar la situación. Uno de los debates más fuertes se dio los días posteriores al juicio que afrontaron varios miembros de la PAH el 26 de enero de 2016, denunciados por la ocupación a Catalunya Caixa. ${ }^{17} \mathrm{La}$ ocupación, que duró tres días (del jueves al sábado), terminó con el desalojo de los activistas encerrados por parte de los cuerpos de seguridad. A continuación, el banco interpuso una denuncia contra los activistas identificados y finalmente los llevó a juicio, con lo que algunos de ellos terminaron condenados a pagar varias multas.

Enfrentarse abiertamente a un juicio, tener que declarar ante el juez y que finalmente cinco de ellos fueran condenados a penas de multas provocó una fuerte disgregación o divergencia en el seno del movimiento. En las asambleas semanales que se sucedieron después de estos hechos, fue un tema recurrente debatir hasta qué punto se debía seguir con la movilización activa. Claramente, esto visibilizó los dos grupos diferenciados que protagonizaron el enfrentamiento de cómo seguir o actuar en futuras acciones.

17. La ocupación tuvo lugar del 12 al 14 de noviembre de 2015, en una de las sedes de Catalunya Caixa en la plaza Sant Joan, en pleno núcleo comercial de la ciudad de Lleida. Esta ocupación se produjo como acción final en la lucha por reclamar soluciones a dos afectadas por hipotecas. 
En este debate, los jóvenes politizados —algunos de ellos con más juicios acumulados por otras acciones en otros marcos reivindicativos - defendieron la necesidad de continuar movilizados, sin preocuparse por la represión, ya que, como argumentaban, se trataba de una causa justa y necesaria. Esto los colocó en una situación hegemónica, por su presencia, persistencia y contundencia tanto en los discursos como en las acciones, lo que provocó un aumento de su poder en la asamblea y, a su vez, un creciente distanciamiento y abandono por parte de otros miembros del grupo que apostaban por el modelo reformista.

Estos cambios internos del movimiento y la consideración hegemónica de un incremento de las acciones como forma de lucha también tuvieron consecuencias directas tanto en el campo político como en el ámbito social y el espacio de los movimientos sociales de la ciudad. Así, el aumento de las acciones y su contundencia tuvo una reacción inmediata en los medios de comunicación y en los discursos políticos institucionales, los cuales criminalizaron el movimiento y lo tildaron de espacio ocupado por jóvenes antisistema violentos. Eso comportó un distanciamiento de la PAH con otros movimientos sociales post-15M y una cierta pérdida del apoyo social en sus acciones (Ballesté, 2018). Pedro explica:

Por ejemplo, ahora circula por allí, que lo van diciendo sobre todo los de Iniciativa [Iniciativa por Catalunya els Verds], que están... Que están furiosos porque ya no tienen el mando allí. ¿No? [Van diciendo] «que la PAH ha sido tomada por antisistemas». Como vamos algunos anarquistas, nosotros y tal... O sea, utilizan el mismo lenguaje de «oh, oh, los peligrosos antisistema» que utilizan en Intereconomía, ¿no? Los de Iniciativa... O sea... ¡En fin!

Con relación a esto y como ejemplo, sobre una acción que tuvo lugar en una oficina del Banco Santander en 2016, los medios de comunicación locales titularon: "Un ejército de mossos desaloja 15 activistas de la PAH de un banco» o «Desalojados a rastras del banco» (información del diario Segre del 4 de marzo de 2016).

\section{De grupos militantes, identificaciones y posiciones políticas}

Para algunos de los teóricos más relevantes sobre los (nuevos) movimientos sociales, la cuestión de la identidad colectiva, los procesos conectivos y la creación de significados comunes de reivindicación frente una situación de adversidad/dominación aparecen como los puntos fundamentales en el entendimiento de la creación de movimientos sociales (Melucci, 1988; Revilla, 1996; Tarrow, 2012; Valenzuela, 2015a). En el presente trabajo se ha mostrado cómo, una vez producidos estos procesos de identidad común en torno a las luchas colectivas — guiadas en este caso por el $15 \mathrm{M}$ y sus movilizaciones posteriores-, posteriormente se dan identificaciones que tienen lugar en el interior de los movimientos y que, lejos de mostrar grupos homogéneos de activistas, manifiestan la pluralidad de atribuciones, significados y prácticas. 
El análisis centrado en los jóvenes activistas participantes de la PAH, con su propia evolución desde el 15M, ha permitido observar las relaciones de poder y de liderazgos que se producen en el seno del movimiento. A su vez, la lucha por hacer hegemónica una forma de entender la protesta y la movilización en cada fase de la PAH ha permitido identificar diferentes relaciones internas, espacios de alianzas y distanciamientos, y resaltar la formación de identificaciones y fronteras entre distintos activistas que se reconocen de forma más o menos situacional en torno a grupos.

El término identificaciones, siguiendo a Brubaker y Cooper (2000), permite alejarnos de la tan amplia concepción de la identidad y heterogeneizar al grupo, y asumir que no se trata de una entidad compacta, hermética e igualitaria, sino que contiene fronteras permeables o difusas entre grupos. Unas fronteras que, en determinados momentos, los agentes superan y traspasan dichas líneas discontinuas (Sánchez y Hakim, 2014; Brubaker y Cooper, 2000).

En todo el proceso descrito de la PAH de Lleida se produce una reorganización interna de los lugares de liderazgo y poder. La aparición de agentes con una visión del activismo más ligada a la acción directa y a la perpetuación de la protesta hasta procurar lograr un cambio de modelo ha provocado la creación de al menos tres grupos manifestados de forma visible tanto en el seno de las asambleas como en la participación de las distintas acciones. Estos grupos tienen distintos postulados sobre cómo debe actuar la PAH y cuál debe ser su objetivo.

Como ya se ha introducido, las dificultades por conseguir mantener a los afectados en la lucha política una vez terminados sus propios casos llevaron a reconsiderar las estrategias de la $\mathrm{PAH}$ — sobre todo en esta nueva fase. En el marco de este debate profundo, acompañado con los acontecimientos que tenían lugar en esas mismas fechas — aumento de las acciones, represión policial y juicios acumulados-, precipitó la aparición/posicionamiento de posturas diferenciadas que se enmarcaban entre el entendimiento de qué acciones se debían llevar a cabo y hasta qué punto mantener el pulso a las entidades bancarias frente a los juicios. Se produjo, utilizando el concepto de Turner, un «drama social» (los juicios y la creciente represión) que provocó la toma de partido de los miembros y facilitó la visibilización de las distintas oposiciones internas que materializaron los grupos enfrentados (Turner, 1986), es decir, las diferentes posiciones en cuanto a los discursos y las prácticas con las que se pretende hacer frente a dicho drama.

El proceso de formación de grupos dentro de la PAH en esta nueva fase, que ahonda sus raíces en otros colectivos y movimientos de la ciudad, permite distinguir entre los que apuestan claramente por la vía de la acción directa y la escalada de fuerza en la lucha por lograr sus objetivos políticos — que normalmente giran en torno a un cambio de modelo profundo-; los que prefieren seguir con la atención a los damnificados por casos hipotecarios - muchos de ellos actualmente por "pobreza energética»—, que normalmente abogan por una confianza mayor en las posibilidades de cambio por la vía institucional —apuestan por una vía de reforma del Estado y sus políticas—; y, por último, los que se mantienen en un punto medio y se balancean entre los dos grupos 
en función de la (su) situación. Estos últimos, con un grado de participación e implicación desigual en las acciones, muestran, según el caso, simpatías por cada grupo en función de sus creencias y situaciones personales.

La consecución de un cierto poder interno por parte de los jóvenes politizados y la asimilación de estrategias y discursos políticos de la PAH próximos a los empleados por los propios colectivos anticapitalistas provocaron una cierta desafección por parte de algunos miembros del colectivo (los afines a la vía reformista). Como consecuencia, por un lado, las asambleas se vieron reducidas en número, a pesar de la creciente asistencia de los jóvenes politizados. Por el otro, el cambio en los discursos, las acciones y las prácticas de las concentraciones, actos y manifestaciones provocó tanto la disminución de la participación de miembros activos de la PAH (vinculado también al aumento de la represión judicial a miembros del movimiento) como la reducción de la solidaridad por parte de activistas de otros movimientos. En ese sentido, durante todo este proceso de recuperación de formatos de acción directa y de inclusión de jóvenes politizados en la $\mathrm{PAH}$, también se produjo de forma paralela una pérdida de centralidad del movimiento dentro del campo político de la ciudad. La menor solidaridad por parte de ciertos políticos institucionales, un aumento de la criminalización en los medios de comunicación locales y el incremento de la presencia policial en sus acciones, así como la represión a través de juicios colectivos o multas, no solo significaron una pérdida de participantes de forma interna, sino también una menor adhesión por parte de otros activistas participantes de movimientos post- $15 \mathrm{M}$ y, también, una invisibilización creciente del movimiento dentro de la ciudad. Esto último se abordará en el siguiente apartado como una de las principales conclusiones de la investigación.

\section{La PAH: de central a periférica}

En el transcurso del período estudiado, y tomando perspectiva desde el 15M, se ha podido ver cómo la PAH en Lleida sufrió un cambio en su interior que se ha manifestado en nuevos roles, liderazgos y relaciones de poder internas. En dicha transformación, los jóvenes participantes han ejercido un papel clave en el proceso por hacer hegemónica una nueva forma de comprender la implicación política del movimiento y las repercusiones sociales y políticas que este debía tener. El poder que suman dichos jóvenes a partir del capital militante acumulado en otros espacios de movilización, visibilizado de forma diáfana en su implicación en las asambleas y acciones, terminó provocando un cambio en las dinámicas internas y también una resituación del movimiento social en el panorama político no institucional de la ciudad.

Las relaciones y los cambios de poder que se vivieron en la PAH durante estos años no solo significaron una modificación de las dinámicas del movimiento, sino que en un sentido amplio también buscaron revertir los mecanismos de movilización que eran hegemónicos desde el 15M. Es decir, buscaron recuperar prácticas políticas como la acción directa o la no presencia de liderazgos para resituar las formas políticas que estos jóvenes siguen en sus propios movimientos. 
Esto supuso la aparición de grupos enfrentados en el seno del movimiento que, en función de la acumulación de poder por parte de cada agente y en relación directa con el capital militante acumulado, hicieron virar las estrategias. Como consecuencia, el incremento de la participación juvenil supuso una ruptura con el modelo asistencialista presente hasta 2013-2014 y la recuperación de la movilización social a través del aumento de las acciones y su contundencia. Todo ello desencadenó un crecimiento de la confrontación que se manifestó en la aparición de juicios colectivos y multas, así como en la presencia del movimiento en los medios de comunicación locales desde una perspectiva negativa.

En el proceso por ampliar las formas de acción y recuperar una protesta conjunta contra el modelo de vivienda y de crédito, escenas como los juicios colectivos o las multas también generaron un cierto distanciamiento interno entre los jóvenes activistas que buscaban aumentar el impacto y seguir con la movilización permanente, y algunos afectados, que, en una situación precaria en lo económico y vital, no siempre podían asumir las consecuencias de dicha movilización.

En ese sentido, la resignificación del movimiento y los cambios de relaciones de poder internas también supusieron un giro del lugar que ocupaba dentro del espacio de los movimientos sociales de la ciudad. Si en los primeros años del post-15M la PAH era uno de los movimientos más activos y con más seguimiento de la ciudad, la continua presión en la movilización de la nueva fase, junto con un incremento de la represión, la trasladaron a un espacio periférico de los movimientos sociales de la ciudad. Las relaciones con otros movimientos disminuyeron, la presencia de políticos institucionales en sus acciones también se redujo y, finalmente, la presencia de activistas solidarios procedentes de movimientos post-15M también se vio alterada.

En definitiva, el presente estudio ha permitido observar la forma en que aquellos jóvenes politizados en el $15 \mathrm{M}$, que posteriormente se involucran en espacios políticos anticapitalistas, se relacionan en la actualidad con los movimientos que surgieron (al menos en Lleida) directamente del propio 15M. A su vez, las relaciones de poder internas, las diferentes acumulaciones de capital militante y la propia evolución del movimiento no solo muestran a la PAH como un espacio en disputa y constante evolución, también permiten comprender cómo esos jóvenes que participaron en el $15 \mathrm{M}$ y luego en la $\mathrm{PAH}$ buscan legitimar de nuevo ciertas prácticas políticas y romper con la hegemonía de las formas políticas indignadas (no violencia, posiciones políticas de consenso y formato de portavoces, entre otras) a través de hacer hegemónicas sus formas dentro del movimiento por la vivienda.

\section{Financiación}

Este artículo ha recibido financiación del European Research Council (ERC) en el marco del programa de Investigación e Innovación de la Unión Europea HORIZON 2020, Grant Agreement No 742705. 


\section{Referencias bibliográficas}

Ballesté, E. (2018). El poder en los movimientos. Jóvenes activistas en el post-15M: estudio de caso sobre la Marea Blanca, la PAH y los Colectivos Anticapitalistas en Lleida. Tesis doctoral. Universitat de Lleida.

- (2019). «Espacios de emancipación: del control biopolítico a la represión. Etnografía de la PAH de Lleida». En: Fraile, P.; Bonastra, Q. y Solís J. M. (eds.). Los contornos del control. Barcelona: Icària.

BARTH, F. (1966). Ethnic Groups and Boundaries. Oslo: Universitetsforlaget.

Bourdieu, P. (1986). «The Forms of Capital». En: Richardson. J. G. (ed.). Handbook of Theory and Research for the Sociology of Education. Nueva York: Greenwood Press.

- (1989). «El espacio social y la génesis de las "clases"». Estudios sobre las Culturas Contemporáneas, 3 (7), 27-55.

Brubaker, R. y Cooper, F. (2000). «Beyond Identity». Theory and Society, 29, 1-47.

Byrne, J. (dir.) (2013). Occupy Wall Street. Barcelona: RBA.

Cabasés, M. A.; Pardell, A. y Feixa, C. (2018). Jóvenes, trabajo y futuro. Perspectivas sobre la garantía juvenil en España y Europa. Valencia: Tirant lo Blanch.

Castells, M. (2015). «Ecos del 15-M». La Vanguardia [en línea]. <http://www.lavanguardia.com/opinion/20151219/30905030774/ecos-del-15-m. html > acceso 2 de mayo 2016.

Claret, A. (2013). Tot el poder als joves! Barcelona: Els Llums.

Colau, A. y Alemany, A. (2013). ¡Sí se puede! Crónica de una pequeña gran victoria. Barcelona: Destino.

Escobar, A. (1995). Encountering development. The making and unmaking of the Third World. Princeton: Princeton University Press.

Feixa, C. (2014). «Revisitación a Gramsci: El Teatro de la Hegemonía». Revista Sinaloense de Ciencias Sociales, 38, 10-23.

FeIXa, C. y Nofre, J. (2013). \#GeneraciónIndignada. Topias y utopias del 15M. Lleida: Milenio.

Feixa, C.; SÁnchez, J. y J. Nofre (2014). «Del altermundismo a la indignación. Cronotopos del activismo político juvenil en Barcelona». Revista Nueva Sociedad, 251, 87-89.

Feixa, C.; Cabases, M. A. y Pardell, A. (2015). «El juvenicidio moral de los jóvenes... Al otro lado del charco». En: Valenzuela, J. M. (coord.). Juvenicidio. Ayotzinapa y las vidas precarias en América Latina y España. Barcelona: NED, 235-269.

Ferrándiz, F. (2011). Etnografías contemporáneas. Anclajes, métodos y claves para el futuro. Barcelona: Anthropos.

Genind (2013-15). La Generación Indignada. Espacio, poder y cultura en los movimientos juveniles de 2011: una perspectiva transnacional. Ministerio de Economía y Competitividad (España). VI Programa Nacional de Investigación Científica, Desarrollo e Innovación Tecnológica, 2008-2011 [CSO2012-34415].

Giugni, M. y Grasso, M. (2016). «The biographical impact of participation in social movement activities: beyond highly committed New Left activism». En: Bosi, L.; Giugni, M. y UbA, K. (eds.). The consequences of Social Movements. Cambridge: Cambridge University Press. <https://doi.org/10.1017/cbo9781316337790.004>

Gledhill, J. (2000). El poder y sus disfraces: perspectivas antropológicas de la política. Barcelona: Bellaterra.

Graeber, D. (2009). Direct Action. An Ethnography. Oakland: AK Press. 
- (2011). Fragmentos de antropología anarquista. Barcelona: Virus.

Gramsci, A. (2013). Antología. Selección, traducción y notas de Manuel Sacristán. Barcelona: Akal.

Hammersley, M. y Atrinson, P. (2014). Etnografia. Métodos de investigación. Barcelona: Espasa.

Juris, J. (2007). «Practicing Militant Ethnography with the Movement for Global Resistance (MRG) in Barcelona». En: Shukaitis, S. y Graeber. D. (eds.). Constituent Imagination, Militant Investigations, Collective Theorization. Oakland: AK Press, 164-176.

Juris, J. y Kashnabish, A. (2013). Insurgent Encounters. Transnational Activism, Ethnography, and the Political. Duke University Press.

Mansilla, J. A. (2015). «Movimientos sociales y apropiaciones colectivas en la Barcelona post-15M: el papel de la Assemblea Social del Poblenou». Etnográfica, 19 (1), 77-97. <https://doi.org/10.4000/etnografica.3909>

Mathieu, L. (2019). «The space of social movements». Social Movement Studies, 1-15. <https://doi.org/10.1080/14742837.2019.1630267>

Matonti, F. y Poupeau, F. (2004). "Le Capital militant. Essai de définition». Actes de la Recherche en Sciences Sociales, 155, 4-11. <https://doi.org/10.3917/arss.155.0004>

Mauger, G. (2003). «Pour une politique réflexive du mouvement social». En: CoursSalies, P. y Vakaloulis, M. (dirs.). Les mobilisations collectives: une controverse sociologique. París: PUF.

Melucci, A. (1988). «Getting Involved: Identity and Mobilization in Social Movements». En: Klandermans, B.; Kriesi, H. y Tarrow. S (eds.). From Structure to Action: Comparing Social Movements Research Across Cultures. International Social Movement Research, 1. Greenwich: Conn., JAI, 329-348.

Molina, J. L. (2001). El análisis de redes sociales. Una introducción. Barcelona: Bellaterra.

Morán, M. L. y Benedicto, J. (2016). «Los jóvenes españoles entre la indignación y la desafección política: Una interpretación desde las identidades ciudadanas». Revista Última Década, 24 (44), 11-38. <https://doi.org/10.4067/s0718-22362016000100002>

NAsH, J. (2008). «Cambios paradigmáticos y dialéctica de los movimientos sociales». Cuadernos de Antropología Social, 28, 7-32.

Navarrete, L. (2011). Desmontando a ni-ni. Un estereotipo juvenil en tiempos de crisis. Madrid: Injuve.

Nofre, J.; Sánchez, J. y Feixa, C. (2015). «Divergences and Divisions in Contemporary Spain's Political Youth Activism». En: Feldman-Barret, C. (ed.). Lost Histories of Youth Culture. Nueva York: Peter Lang, 217-236.

PAstor, J. (2013). «El 15M, las Mareas y su relación con la política sistémica. El caso de Madrid». Anuari del Conflicte Social, 2013, 224-247.

Pleyers, G. (2011). Alter-globalization. Becoming Actors in the Global Age. Malden: Polity Press.

- (2013). «From Local Ethnographies to Global Movement. Experience, Subjectivity, and Power among Four Alter-globalization actors». En: Juris, J. y KashnABISH, A. (eds.). Insurgent Encounters. Transnational Activism, Ethnography, and the Political. Duke University Press, 108-128. <https://doi.org/10.1515/9780822395867-007> 
Porta, D. della y Diani, M. (2011). Los movimientos sociales. Madrid: Complutense. RAzQuin, A. (2016). «Erosión de la participación ciudadana en la movilización social: Cierres organizativos y repliegues sobre el capital escolar». Empiria, 33, 65-87. <https://doi.org/10.5944/empiria.33.2016.15864>

Revilla, M. (1996). «El concepto de movimiento social: acción, identidad y sentido». Revista Última Década (5), 1-18.

Sánchez, J. y Hakim, N. (2014). «¿Qué significa ser joven? Reflexión teórica desde dos ejemplos etnográficos». Quaderns-e de l'Institut Català d'Antropologia, 19 (2), 43-57.

Scheper-Hughes, N. (1995). «The Primacy of the Ethical: Propositions for a Militant Anthropology». Current Anthropology, 36 (3), 409-440. <https://doi.org/10.1086/204378>

Sсотт, J. (1985). Weapons of the Weak: Everyday Forms of Peasant Resistance. Yale: Yale University Press.

Strecker, T.; Ballesté, E. y Feixa, C. (2018). «El juvenicidio moral en España: antecedentes del concepto, causas y efectos». En: Cabasés, M. A.; Pardell, A. y FeiXA, C. (eds.). Jóvenes, trabajo y futuro. Valencia: Tirant lo Blanch.

Subirats, J. (2015). «Todo se mueve. Acción colectiva, acción conectiva. Movimientos, partidos e instituciones». Revista Española de Sociología, 24, 123-131.

TаIво, C. (2013). "The Spanish Indignados: A movement with two souls». European Urban and Regional Studies, 20 (1), 155-158. <https://doi.org/10.1177/0969776412459846>

TARrow, S. (2012). El poder en movimiento. Los movimientos sociales, la acción colectiva y la política. Madrid: Alianza.

Tilly, C. (2005). Identities, boundaries and social ties. Londres: Paradigm Publishers. <https://doi.org/10.4324/9781315634050>

Tilly, C. y Wood, L. J. (2010). Los movimientos sociales, 1768-2008. Desde sus orígenes a Facebook. Barcelona: Crítica.

Tugas, R. (2014). Escac al poder. L'auge de l'esquerra alternativa. Barcelona: Deu i Onze.

Turner, V. (1986). Del rito al teatro. Bolonia: Il Mulino.

Valenzuela, J. M. (2012). Sed de mal. Tijuana: El Colegio de la Frontera Norte.

- (2015a). El sistema es antinosotros. Culturas, movimientos y resistencias juveniles. Tijuana: El Colegio de la Frontera Norte.

- (2015b). Juvenicidio. Ayotzinapa y las vidas precarias en América Latina y España. Tijuana: El Colegio de la Frontera Norte.

Vommaro, P. (2015). Juventudes y políticas en la Argentina y en América Latina. Tendencias, conflictos y desafíos. Ciudad Autónoma de Buenos Aires: Grupo Editor Universitario. <https://doi.org/10.2307/j.ctvtxw3h3>

Weber, M. (2007). Sociología del poder: los tipos de dominación. Madrid: Alianza.

Yates, K. (2015). «Rethinking Prefiguration: Alternatives, Micropolitics and Goals in Social Movements». Social Movement Studies, 14 (1), 1-21. <https://doi.org/10.1080/14742837.2013.870883> 